PROCEEDINGS OF THE

AMERICAN MATHEMATICAL SOCIETY

Volume 127, Number 6, Pages 1697-1703

S 0002-9939(99)04663-8

Article electronically published on February 11, 1999

\title{
COMPOSITION OPERATORS: HYPERINVARIANT SUBSPACES, QUASI-NORMALS AND ISOMETRIES
}

\author{
BRUCE A. CLOAD
}

(Communicated by Theodore W. Gamelin)

\begin{abstract}
We exhibit hyperinvariant subspaces of some composition operators. We also consider quasi-normal composition operators and discuss the commutant of isometric composition operators.
\end{abstract}

\section{INTRODUCTION}

Let $D$ be the unit disc in the complex plane. The Hardy space on $D, H^{2}(D)$, is defined to be the set of analytic functions on $D$ which have square summable power series coefficients. Given an analytic self map of the disc, $\phi$, we may define a composition operator $C_{\phi}$ on $H^{2}$ by $C_{\phi}(f)=f \circ \phi$, for all $f$ in $H^{2}$. These bounded operators have been studied extensively (see [18] or [10]).

We say an operator $B$ commutes with an operator $A$ if $A B=B A$. Recently, we have been interested in which operators $B \in B\left(H^{2}\right)$ commute with a given composition operator, $C_{\phi}$ (see [6] and [7]). In the second section we address the question of what are the hyperinvariant subspaces for a composition operator, $C_{\phi}$, induced by a particular type of function, $\phi$. The question was of interest in that a solution may provide a tool for classifying the commutant of $C_{\phi}$; that is, the algebra of all operators which commute with $C_{\phi}$. In particular, in the second section, we show (Corollary 2) that if $C_{\phi}$ is Riesz, it has a triangularizing chain of hyperinvariant subspaces. In the third section, we develop some tools for classifying quasi-normal composition operators, while in the fourth section, we discuss the commutant of composition operators which are also isometries and pose some research questions.

\section{HYPERINVARIANT SUBSPACES}

Let $\phi$ be an analytic self map of the disc and define

$$
\phi^{[n]}=\underbrace{\phi \circ \phi \circ \ldots \circ \phi}_{n \text { times }},
$$

Received by the editors May 13, 1997 and, in revised form, September 8, 1997.

1991 Mathematics Subject Classification. Primary 47B38; Secondary 47A15, 47B06, 47B20.

The results in this paper are part of the author's doctoral thesis under the direction of Peter Rosenthal at the University of Toronto. The author would like to thank NSERC for their support as well as the referee for his kind comments and helpful suggestions.

(C)1999 American Mathematical Society 
the $n^{\text {th }}$ iterate of $\phi$ under composition. Also, suppose $\phi(0)=0$ and $0<\left|\phi^{\prime}(0)\right|<1$. In 1884, Koenigs showed that the sequence $\left\{\sigma_{k}\right\}$ with

$$
\sigma_{k}(z)=\frac{\phi^{[k]}(z)}{\left(\phi^{\prime}(0)\right)^{k}}
$$

converges uniformly on compact subsets of $D$ to a non-constant function $\sigma$, which is known as the Koenigs' function for $\phi$ (see [18] or [10]). Paul Bourbon proved the following theorem when $\phi$ was univalent, and shortly afterwards Pietro PoggiCorradini was able to remove that hypothesis (see [1] or [14]).

Theorem 1. Let $\phi$ be an analytic self map of the disc with $\phi(0)=0$ and $0<$ $\left|\phi^{\prime}(0)\right|<1$. Let $\sigma$ be the Koenigs' function of $\phi$ and $q$ a natural number. If $(\sigma)^{q}$ is in $H^{2}$, then the sequence $\left\{\left(\sigma_{k}\right)^{q}\right\}$ converges to $(\sigma)^{q}$ in the $H^{2}$ norm.

In [2], Bourdon and Shapiro proved a sufficient condition for the Koenigs' function to belong to $H^{p}$ and showed the condition to be necessary in the case that the function $\phi$ is analytic on the closed unit disc. In [15], Pietro Poggi-Corradini was able to prove the necessity of the condition without any additional conditions on $\phi$. These results together lead to the following theorem.

Theorem 2. Let $\phi$ be an analytic self map of the disc with $\phi(0)=0$ and $0<$ $\left|\phi^{\prime}(0)\right|<1$. Let $\sigma$ be the Koenigs function. Then $(\sigma)^{q}$ is in $H^{2}$ if and only if $\left|\phi^{\prime}(0)\right|^{q}$ exceeds the essential spectral radius of $C_{\phi}$.

A subspace $M$ is hyperinvariant for an operator $A$ if it is invariant for every operator which commutes with $A$.

Theorem 3. Let $\phi$ be an analytic self map of the disc with $\phi(0)=0$ and $0<$ $\left|\phi^{\prime}(0)\right|<1$. Suppose $(\sigma)^{q}$ is in $H^{2}$ where $\sigma$ is the Koenigs' function of $\phi$. Then for each natural number $k, 1 \leq k \leq q$, the subspaces $z^{k} H^{2}$ are hyperinvariant for $C_{\phi}$.

Proof. In [7], we showed that $z H^{2}$ is a hyperinvariant subspace for such a $C_{\phi}$. This covers the case $k=1$. Proceeding by induction on $n<q$, we will assume, for $k \leq n$, that $z^{k} H^{2}$ is hyperinvariant for $C_{\phi}$. Let $A$ be an operator which commutes with $C_{\phi}$ and let $z^{n+1} p$ be a function in $z^{n+1} H^{2}$ where $p$ is a polynomial. We wish to show

$$
\left\langle A\left(z^{n+1} p\right), z^{l}\right\rangle=0
$$

for $l \leq n$. Since $z^{n+1} p$ is in $z^{n} H^{2}$, we have by the induction hypothesis that

$$
\left\langle A\left(z^{n+1} p\right), z^{l}\right\rangle=0
$$

for $l<n$. Now

$$
\begin{gathered}
\left\langle A C_{\phi}^{m}\left(z^{n+1} p\right), z^{n}\right\rangle=\left\langle A\left(z^{n+1} p\right),\left(C_{\phi}^{*}\right)^{m}\left(z^{n}\right)\right\rangle \\
=\left\langle A\left(z^{n+1} p\right),\left(\overline{\phi^{\prime}(0)}\right)^{m n} z^{n}\right\rangle .
\end{gathered}
$$

The last equality follows from the induction hypothesis and the fact that $C_{\phi}^{*}$ is upper triangular as a matrix when represented in the standard basis. Dividing both sides of the equation by $\left(\phi^{\prime}(0)\right)^{m n}$, it follows that

$$
\left\langle A\left(\left(\sigma_{m}\right)^{n} \phi^{[m]}\left(p \circ \phi^{[m]}\right)\right), z^{n}\right\rangle=\left\langle A\left(z^{n+1} p\right), z^{n}\right\rangle .
$$

The left-hand side of this equation is equal to

$$
\left\langle\left(\sigma_{m}\right)^{n} \phi^{[m]}\left(p \circ \phi^{[m]}\right), A^{*}\left(z^{n}\right)\right\rangle .
$$


Since $H^{2 q} \subset H^{2 n}$ when $q \geq n,(\sigma)^{n}$ is in $H^{2}$. By Theorem $1,\left(\sigma_{m}\right)^{n}$ is a bounded sequence in the $H^{2}$ norm. Since $p$ is in $H^{\infty}$,

$$
\left\{\left(\sigma_{m}\right)^{n} \phi^{[m]}\left(p \circ \phi^{[m]}\right)\right\}
$$

is also a bounded sequence in the $H^{2}$ norm. This sequence converges pointwise to 0 as $m$ goes to $\infty$, and thus it converges weakly to 0 . It follows that $\left\langle A\left(z^{n+1} p\right), z^{n}\right\rangle=$ 0.

If $z^{n+1} f$ is in $z^{n+1} H^{2}$, we may find a sequence of polynomials $\left\{p_{l}\right\}$ which converges strongly to $f$ as $l$ goes to $\infty$. In particular, $\left\langle A\left(z^{n+1} p_{l}\right), z^{n}\right\rangle$ will converge to $\left\langle A\left(z^{n+1} f\right), z^{n}\right\rangle$ as $l$ goes to infinity. Hence the result holds.

Let $\|A\|_{e}$ denote the essential norm of an operator $A$. An operator $A$ is a Riesz operator if $\left\|A^{n}\right\|_{e}^{\frac{1}{n}}$ tends to 0 as $n$ tends to $\infty$. This implies that $A$ is Riesz if and only if the essential spectrum of $A$ is $\{0\}$. In [3], Bourdon and Shapiro show that a Riesz composition operator must be induced by a function which has an interior fixed point in $D$. For basic details of Riesz operators see [11].

Corollary 1. Let $\phi$ be an analytic self map of $D$ with $\phi(b)=b$ where $b$ is in $D$ and $0<\left|\phi^{\prime}(b)\right|<1$. If $C_{\phi}$ is a Riesz operator, then $C_{\phi}$ has a triangularizing chain of hyperinvariant subspaces.

Proof. Let $\alpha(z)=\frac{b-z}{1-\bar{b} z}$. Define $\psi=\alpha \circ \phi \circ \alpha$. Note $\psi(0)=0$ and $0<\left|\psi^{\prime}(0)\right|<1$. Since $C_{\psi}^{n}=C_{\alpha} C_{\phi}^{n} C_{\alpha}$, it follows that $C_{\psi}$ is a Riesz operator. Now by Theorem 2 and Theorem $3, C_{\psi}$ has the $z^{q} H^{2}$ as hyperinvariant subspaces for all $q=0,1,2 \ldots$. Thus $C_{\phi}$ has $(\alpha)^{q} H^{2}, q=0,1,2 \ldots$ as a chain of hyperinvariant subspaces. Moreover, any operator which holds these subspaces invariant must be lower triangular with respect to the orthonormal basis $\left\{(\alpha)^{n} \frac{1-|b|^{2}}{1-\bar{b} z}: n=0,1,2 \ldots\right\}$.

Let $f^{(k)}$ be the $k^{\text {th }}$ derivative of $f$.

Corollary 2. Let $\phi$ be an analytic self map of the disc with $\phi(0)=0$ and $0<$ $\left|\phi^{\prime}(0)\right|<1$. Let $k$ be a natural number greater than or equal to 1 . Suppose $(\sigma)^{k+1}$ is in $H^{2}$ and let $A$ commute with $C_{\phi}$. Then, for all $f$ in $H^{2}, \frac{d^{k}}{d z^{k}}(A(f))(0)=$ $k ! \sum_{n=1}^{k} \frac{f^{(n)}(0)}{n !}\left\langle A\left(z^{n}\right), z^{k}\right\rangle$.

Proof. Let $f=\sum_{n=0}^{\infty} a_{n} z^{n}$ be the power series expansion of $f$. In [7], we showed that if $A$ commutes with $C_{\phi}$, then $(A(f))(0)=A(1) f(0)$ where $A(1)$ is a constant. This implies that $\left\langle A\left(a_{0}\right), z^{k}\right\rangle=0$. Now

$$
\left\langle A(f), z^{k}\right\rangle=\left\langle A\left(a_{0}\right), z^{k}\right\rangle+\sum_{n=1}^{k} a_{n}\left\langle A\left(z^{n}\right), z^{k}\right\rangle+\left\langle A\left(\sum_{n=k+1}^{\infty} a_{n} z^{n}\right), z^{k}\right\rangle .
$$

The last term is 0 since by Theorem $3, z^{k+1} H^{2}$ is hyperinvariant for $C_{\phi}$. Since $a_{n}=\frac{f^{(n)}(0)}{n !}$, the result follows.

The next example shows that there are analytic self maps of $D$ with $\phi(0)=0$ and $\phi^{\prime}(0)=0$ such that the $z^{q} H^{2}$ are not hyperinvariant for $C_{\phi}$ for $q \geq 2$.

Example 1. Let $n$ be a natural number greater than 1. For $C_{z^{n}}$, the subspaces $z^{q} H^{2}$ for $q \geq 2$ are not hyperinvariant. 
Proof. In [6], we discuss the commutant of $C_{z^{n}}$ in more detail. Let $q$ be a natural number greater than or equal to 2 . If we define an operator $A$ on $H^{2}$ by $A\left(z^{q \cdot n^{l}}\right)=$ $z^{n^{l}}$ and $A\left(z^{k}\right)=0$ when $k \neq q \cdot n^{l}$, then $A$ commutes with $C_{\phi}$. Now $A\left(z^{q}\right)=z$ and hence $z^{q} H^{2}$ is not a hyperinvariant subspace for $C_{\phi}$.

\section{QUASI-NORMALS}

We now turn to quasi-normal operators. An operator $A$ is quasi-normal if $A$ commutes with $A^{*} A$. For more information, see [8].

Lemma 1. If $C_{\phi}$ is quasi-normal, then $\phi(0)=0$.

Proof. $C_{\phi} C_{\phi}^{*} C_{\phi}(1)=C_{\phi}^{*} C_{\phi} C_{\phi}(1)$ implies that

$$
\frac{1}{1-\overline{\phi(0)} \phi(z)}=\frac{1}{1-\overline{\phi(0)} z} \text {. }
$$

It follows that $\phi(0)=0$.

Lemma 2. Let $\phi$ belong to $L^{2}(\partial D)$ with $\phi$ non-zero and not a characteristic function of a proper subset of the unit circle. Also suppose that $\|\phi\|_{L^{2}}=\mu$ and $\left\|(\phi)^{k_{l}}\right\|_{L^{2}}=\mu$ where $k_{l}$ is a sequence of natural numbers which diverges to $\infty$ and $\mu$ is a non-zero real number. Then $\left|\phi\left(e^{i \theta}\right)\right|=1$ almost everywhere on $\partial D$.

Proof. Let $\lambda=\mu^{2}$. Let $m(A)$ be the normalized standard Lebesgue measure of a set $A \subset \partial D$. Suppose that $\left|\phi\left(e^{i \theta}\right)\right|>1$ on a set of positive measure of $\partial D$. Then, in particular, there exists $\epsilon>0$ such that the set $A_{\epsilon}=\left\{e^{i \theta}:\left|\phi\left(e^{i \theta}\right)\right|>1+\epsilon\right\}$ has positive measure. It follows that

$$
\lambda=\left\|\phi^{k_{l}}\right\|_{L^{2}}^{2} \geq \frac{1}{2 \pi} \int_{A_{\epsilon}}|\phi(z)|^{2 k_{l}} d z \geq(1+\epsilon)^{2 k_{l}} m\left(A_{\epsilon}\right) .
$$

As $k_{l}$ tends to infinity, the right-hand side also tends to infinity. This is a contradiction; thus $\left|\phi\left(e^{i \theta}\right)\right| \leq 1$ almost everywhere.

Similarly, suppose that $\left|\phi\left(e^{i \theta}\right)\right|<1$ on a set of positive measure of $\partial D$. Then, in particular, there exists $\epsilon>0$ such that the set $B_{\epsilon}=\left\{e^{i \theta}:\left|\phi\left(e^{i \theta}\right)\right|<1-\epsilon\right\}$ has positive measure. Then

$$
\lambda=\frac{1}{2 \pi} \int_{B_{\epsilon}}|\phi(z)|^{2} d z+\frac{1}{2 \pi} \int_{\partial D \backslash B_{\epsilon}}|\phi(z)|^{2} d z .
$$

Let $\int_{\partial D \backslash B_{\epsilon}}|\phi(z)|^{2} d z=\kappa$. We note $\kappa$ is strictly less than $\lambda$. Now

$$
\frac{1}{2 \pi} \int_{B_{\epsilon}}|\phi(z)|^{2 k_{l}} d z \leq(1-\epsilon)^{2 k_{l}} m\left(B_{\epsilon}\right) .
$$

Choose a natural number $N$ such that $k_{l} \geq N$ implies that $(1-\epsilon)^{2 k_{l}} m\left(B_{\epsilon}\right)$ is strictly less than $\lambda-\kappa$. Since $|\phi(z)| \leq 1$ almost everywhere, we note that $|\phi(z)|^{k_{l}} \leq|\phi(z)|$. Then with $k_{l} \geq N$,

$$
\begin{gathered}
\lambda=\left\|(\phi)^{k_{l}}\right\|_{L^{2}}^{2}=\frac{1}{2 \pi} \int_{B_{\epsilon}}|\phi(z)|^{2 k_{l}} d z+\frac{1}{2 \pi} \int_{\partial D \backslash B_{\epsilon}}|\phi(z)|^{2 k_{l}} d z \\
\leq \frac{1}{2 \pi} \int_{B_{\epsilon}}|\phi(z)|^{2 k_{l}} d z+\frac{1}{2 \pi} \int_{\partial D \backslash B_{\epsilon}}|\phi(z)|^{2} d z
\end{gathered}
$$




$$
<\lambda-\kappa+\frac{1}{2 \pi} \int_{\partial D \backslash B_{\epsilon}}|\phi(z)|^{2} d z=\lambda
$$

which is a contradiction; thus $|\phi(z)|=1$ almost everywhere.

Theorem 4. Suppose $C_{\phi}$ is quasi-normal. If $C_{\phi}^{*} C_{\phi}$ is a diagonal matrix in the standard basis, then either $\phi(z)=c z$ for some constant $c$ of modulus less than 1 or $\phi$ is an inner function.

Proof. Let the power series of $\phi$ be $\sum_{n=1}^{\infty} a_{n} z^{n}$ and let the $k^{\text {th }}$ diagonal entry of $C_{\phi}^{*} C_{\phi}$ be $\lambda_{k}$. First of all, if $\phi$ is identically zero or if only one of the $a_{n}$ is non-zero, then we are done.

Next suppose that only a finite number of the coefficients $a_{n}$ are non-zero but more than one of them is non-zero. Thus let $\phi(z)=z^{k} a_{k}+\cdots+a_{l} z^{l}$ where $a_{l}$ and $a_{k}$ are non-zero and $k$ is the smallest power of $z$ with a non-zero coefficient and $l$ is the greatest. Now,

$$
\left\langle(\phi(z))^{l},(\phi(z))^{k}\right\rangle=\left(a_{k}\right)^{l}\left(a_{l}\right)^{k},
$$

which by hypothesis must be zero. Thus if only a finite number of coefficients are non-zero, we have $\phi(z)=c z^{k}$ where $c$ is a constant of modulus less than or equal to 1 . If $k \geq 2$ and $c$ is less than 1 in modulus, then $C_{\phi}$ is not quasi-normal, so in this case $k=1$.

Suppose an infinite number of the coefficients $a_{n}$ are non-zero. Then $\phi(z)=$ $\sum_{n=k}^{\infty} a_{n} z^{n}$ where $a_{k}$ is the first non-zero coefficient. Now $C_{\phi}^{*} C_{\phi} C_{\phi}(z)=C_{\phi} C_{\phi}^{*} C_{\phi}(z)$ implies that

$$
\sum_{n=k}^{\infty} \lambda_{n} a_{n} z^{n}=\sum_{n=k}^{\infty} \lambda_{1} a_{n} z^{n}
$$

It follows that $\lambda_{n}=\lambda_{1}$ for infinitely many $n$. Since $\lambda_{1}=\langle\phi(z), \phi(z)\rangle$ and $\lambda_{n}=$ $\left\langle(\phi(z))^{n},(\phi(z))^{n}\right\rangle$, we may apply Lemma 2 to conclude that $\phi$ must be an inner function.

Corollary 3. If $C_{\phi}$ has $z^{q} H^{2}, 1 \leq q<\infty$, as hyperinvariant subspaces, and $C_{\phi}$ is quasi-normal, then either $\phi(z)=c z$ for some $c$ of modulus less than 1 or $\phi$ is inner.

Proof. If $C_{\phi}$ is quasi-normal, then $C_{\phi}$ commutes with $C_{\phi}^{*} C_{\phi}$, as does $C_{\phi}^{*}$. Thus $C_{\phi}^{*} C_{\phi}$ is both upper and lower triangular with respect to the standard basis and thus diagonal. Apply Theorem 4.

We could apply Corollary 1 to conclude that if $C_{\phi}$ is quasi-normal and Riesz, then $\phi(z)=c z$ for $\|c\|<1$. This would say that if $C_{\phi}$ is Riesz and quasi-normal, then it is normal. As the next theorem shows, this is true in greater generality.

Theorem 5. If $A$ is an operator on a Hilbert space $H$, and $A$ is Riesz and quasinormal, then $A$ is normal.

Proof. If $A$ is identically 0 , then we are done. Assume $A$ is not equal to 0 . By [4], $\operatorname{ker}(A)$ is reducing, so we may decompose $A$ as $A_{1} \bigoplus 0$ with $A_{1}$ quasi-normal and injective. Moreover, we may decompose $A_{1}$ as $B V$ with $B$ self-adjoint and injective, $V$ isometric, and $B$ commuting with $V$. Since $A_{1}^{n}=V^{n} B^{n}$, we have $\left\|\left(V^{*}\right)^{n} A_{1}^{n}\right\|=\left\|B^{n}\right\|$. Thus

$$
\left\|B^{n}\right\|_{e}^{\frac{1}{n}} \leq\left\|\left(V^{*}\right)^{n}\right\|_{e^{\frac{1}{n}}}^{\frac{1}{n}}\left\|A_{1}^{n}\right\|_{e^{\frac{1}{n}}} \leq\left\|A_{1}^{n}\right\|_{e^{\frac{1}{n}}}^{n} .
$$


The last term goes to 0 as $n$ goes to $\infty$, and thus $B$ is also Riesz. By Theorem 3.7 in [19], since $B$ is self-adjoint and Riesz, it must be compact. Hence by the spectral theorem, $B$ is unitarily equivalent to $\sum \bigoplus \lambda_{k} I_{k}$ where $\lambda_{k}$ are the non-zero eigenvalues and the $I_{k}$ are identity operators on finite-dimensional spaces. Since eigenspaces are hyperinvariant, and $V$ commutes with $B, V$ is unitarily equivalent to $\sum \bigoplus V_{k}$, where the $V_{k}$ are isometries on finite-dimensional spaces and hence unitary. Thus $V$ is a unitary operator and $A_{1}$ is a product of commuting normals and thus normal.

\section{ISOMETRIES}

An operator $A$ on $H^{2}$ is an isometry if $A^{*} A=I$. In [17], Schwarz proves the following theorem.

Theorem 6. $C_{\phi}$ is an isometry on $H^{2}$ if and only if $\phi(0)=0$ and $\phi$ is an inner function.

If $\phi$ is an elliptic disc automorphism, then the commutant of $C_{\phi}$ is well understood (see [6] or [7]). If $\phi$ is not an elliptic disc automorphism and $C_{\phi}$ is an isometry, Nordgren ([13]) shows that $C_{\phi}$, restricted to the constants, and $C_{\phi}$, restricted to $z H^{2}$, are the unitary and purely isometric parts, respectively. If $A$ commutes with $C_{\phi}$, then the constants are a reducing subspace for $A$ (see [7]). Hence we may consider the commutant of $C_{\phi}$ as the direct sum of the commutant of the unitary part and the commutant of the purely isometric part. The purely isometric part is similar to a unilateral shift of infinite multiplicity on the wandering subspace, $M$, of $C_{\phi}$ (see [16]). The commutant of such a unilateral shift is given in terms of multiplication operators on $H^{2}(M)$ (see [16]).

Lemma 3. Let $\phi=z f$ where $f$ is a nonconstant inner function. Let $\left\{g_{n}: n=\right.$ $0,1, \ldots\}$ be an orthogonal basis for $\left(f H^{2}\right)^{\perp}$. Then the following set, $S$,

$$
\left\{z(\phi)^{k} g_{n}: n=0,1,2 \ldots, k=0,1,2 \ldots\right\},
$$

is an orthogonal basis for the wandering subspace of $C_{\phi}$ on $H^{2}$.

Proof. It is easy to see that the vectors in $S$ are orthogonal. The wandering subspace is given by the perp of $C_{\phi}\left(z H^{2}\right)$ in $z H^{2}$ (see [12]). We want to show that the vectors in $S$ span this space. We note that the set of vectors $T$, given by $\left\{(\phi)^{k}: k=1,2, \ldots\right\}$ span $C_{\phi}\left(z H^{2}\right)$. Thus it is sufficient to show that the vectors in $T$ and $S$, along with the set containing the constant function 1, span all of $H^{2}$ since these three sets are mutually orthogonal.

Suppose that $h$ is orthongal to the vectors in these three sets. We claim that $h$ is 0 . First of all $\langle h, 1\rangle=0$ implies that $h=z k_{1}$ for some $k_{1}$ in $H^{2}$. Now

$$
\left\langle z k_{1}, z g_{n}\right\rangle=\left\langle k_{1}, g_{n}\right\rangle=0
$$

for all $n$ implies that $k_{1}$ belongs to $f H^{2}$. Thus $h=z f h_{1}$ for some $h_{1}$ in $H^{2}$. Thus $h=\phi h_{1}$. Now if we proceed by induction and assume that $h=(\phi)^{l} h_{l}$ for some $h_{l}$ in $H^{2}$, we have that $\left\langle(\phi)^{l} h_{l},(\phi)^{l}\right\rangle=0$ implies that $h_{l}=z k_{l+1}$ for some $k_{l+1}$ in $H^{2}$. Thus

$$
\left\langle(\phi)^{l} z k_{l+1},(\phi)^{l} z g_{n}\right\rangle=\left\langle k_{l+1}, g_{n}\right\rangle=0
$$

for all $n$ implies that $k_{l+1}=f h_{l+1}$ for some $h_{l+1}$ in $H^{2}$. Thus $h=(\phi)^{l+1} h_{l+1}$ and we have by induction that $(\phi)^{l}$ divides $h$ for arbitrary powers of $l$ and hence $h=0$. 
In particular, let $\phi=z B$ where $B$ is a Blaschke product with zero set $\left\{a_{n}\right.$ : $n=0,1,2 \ldots\}$. Let $k_{\lambda}$ be the reproducing kernel function for $\lambda$ in $D$ and let $B_{l}$ be the Blaschke product with zero set $\left\{a_{n}: n=0,1, \ldots, l\right\}$. Then the following is an orthonormal basis for $\left(B H^{2}\right)^{\perp}:\left\{g_{0}=k_{a_{0}}, g_{1}=B_{0} k_{a_{1}}, \ldots, g_{n}=B_{n-1} k_{a_{n}}\right\}$. This gives us a basis for the wandering subspace of $C_{\phi}$ and the commutant of $C_{\phi}$ can be explicitly interpreted in terms of this basis.

\section{Questions}

(1): If $\phi$ is an analytic self map of $D, \phi(0)=0$ and $0<\left|\phi^{\prime}(0)\right|<1$, are the subspaces $\left\{z^{q} H^{2}\right\}$ hyperinvariant subspaces for all natural numbers $q$ ? If this is true, then the only $C_{\phi}$ which are quasi-normal are either isometries or are given by $\phi(z)=c z$ for some constant $c$ of absolute value less than 1 .

(2): If $\phi$ is an analytic self map of $D, \phi(0)=0$ and $\phi^{\prime}(0)=0$, what can be said about the hyperinvariant subspaces of $C_{\phi}$ ? If $C_{\phi}$ is quasi-normal, what form does $\phi$ have to take?

(3): Given a basis for the wandering subspace of an isometric composition operator, can the hyperinvariant subspaces or the composition operators which commute be explicitly determined?

\section{REFERENCES}

[1] P.S. Bourdon, Convergence of the Koenigs' Sequence, preprint.

[2] P.S. Bourdon \& J. Shapiro, Mean Growth of Koenigs' Eigenfunctions, J. Amer. Math. Soc. 10 (1997), 299-325. MR 97h:30040

[3] P.S. Bourdon \& J. Shapiro, Riesz Composition Operators, Preprint.

[4] A. Brown, On a Class of Operators, Proc. Amer. Math. Soc. 4, 723-728. MR 15:538c

[5] R.B. Burckel, Iterating Analytic Self-Maps of Discs, Amer. Math. Monthly 88(1981), 396-407. MR 82g:30046

[6] B. Cload, Generating the Commutant of a Composition Operator, Cont. Math. 239 (1998), $11-15$.

[7] B. Cload, Toeplitz Operators in the Commutant of a Composition Operator, to appear in Studia Math.

[8] J.B. Conway, Subnormal Operators, Boston: Pitman Adv. Pub. Program, 1981. MR 83i: 47030

[9] C.C. Cowen, Commuting Analytic Functions, Trans. Amer. Math. Soc. 265(1981), 69-95. MR 85i:30054

[10] C.C. Cowen \& B. MacCluer, Composition Operators on Spaces of Analytic Functions, CRC Press, 1995. MR 97i:47056

[11] H. Dowson, Spectral Theory of Linear Operators, Academic Press, 1978 MR 80c:47022

[12] P.R. Halmos, Shifts on Hilbert Spaces, J, Reine Angew. Math., 208(1961), 102-112. MR 27:2868

[13] E.A. Nordgren, Composition Operators, Canadian J. Math. 20(1968), 442-449. MR 36:6961

[14] P. Poggi-Corradini, Hardy Norm Convergence of the Koenigs' sequence for non-univalent maps, preprint.

[15] P. Poggi-Corradini, The Hardy Class of Koenigs maps, preprint.

[16] H. Radjavi \& P. Rosenthal, Invariant Subspaces, Springer-Verlag (1973). MR 51:3924

[17] H. Schwarz, Composition Operators on $H^{p}$, Ph.D. Thesis, University of Toledo, 1969.

[18] J.H. Shapiro, Composition Operators and Classical Function Theory, Springer-Verlag, New York, 1993. MR 94k:47049

[19] T.T. West, The Decomposition of Riesz Operators, Proc. London Math. Soc. 3(1966), 131140. MR 33:6417

Department of Mathematics, Brock University, St. Catharines, Ontario, Canada L2S 3A1

E-mail address: bcload@spartan.ac.brocku.ca 